\title{
PRÁTICAS DE EDUCAÇÃO EM SAÚDE EM DOMICÍLIO: A EXTENSÃO UNIVERSITÁRIA COMO CENÁRIO DE INTERVENÇÕES A UM INDIVÍDUO HIPERTENSO
}

\author{
Alana Betina Dick, Andréia Ghiggi, Claudete Fátima Hofstetter, Shaiane Ávila da Silva, \\ Suélen Fernanda Schneider, Arlete Eli Kunz da Costa, Luís Felipe Pissaia
}

Resumo: Este estudo possui o objetivo de verificar a instrumentalização de práticas de educação em saúde durante visitas domiciliares a um indivíduo com Hipertensão Arterial Sistêmica por meio de um projeto de extensão universitária. Trata-se de um estudo de caso, intervencional e com abordagem qualitativa, realizado com base em um participante do projeto "Ações Interdisciplinares de Cuidado em Saúde", desenvolvido pela UNIVATES, que mantém suas ações junto a um bairro no município de Lajeado/ RS. Na análise de informações, surgiram três linhas intervencionais, sendo a primeira sobre os hábitos alimentares na qual se verificou que participante ingere alimentos saudáveis, no entanto mantém um consumo elevando de sal na dieta. $\mathrm{Na}$ segunda, trabalhou-se com a prática de exercícios físicos através de alongamentos e exercícios, relatando melhoria na qualidade do sono, diminuição de dor nos membros inferiores e motivação para combater o seu sedentarismo. E na terceira linha de intervenção sobre a adesão ao tratamento medicamentoso, tratou buscar alternativas para organização das medicações utilizadas, com finalidade de facilitar sua adesão ao tratamento, sendo bem aceito pelo participante. Considera-se que as ações de educação em saúde realizadas junto ao indivíduo foram capazes de oferecer uma maior qualidade de vida e controle da doença.

Palavras-chave: Hipertensão Arterial Sistêmica. Visita Domiciliar. Extensão Universitária. Qualidade de Vida.

\section{HOME HEALTH EDUCATION PRACTICES: THE UNIVERSITY EXTENSION AS AN INTERVENTION SCENARIO FOR A HYPERTENSIVE INDIVIDUAL}

\begin{abstract}
This study aims to verify the instrumentalisation of health education practices during home visits to an individual with Systemic Arterial Hypertension through a university extension project. It is a case study, interventional and qualitative approach, based on a participant in the project "Interdisciplinary Actions of Health Care", developed by UNIVATES, which maintains its actions next to a neighborhood in the municipality of Lajeado / RS. In the information analysis, three intervention lines appeared, being the first one on the eating habits in which it was verified that participant ingests healthy foods, nevertheless maintains an elevated consumption of salt in the diet. In the second, we worked with the practice of physical exercises through stretching and exercises, reporting improvement in sleep quality, decreased pain in the lower limbs and motivation to combat their sedentary lifestyle. And in the third line of intervention on adherence to drug treatment, sought to seek alternatives to organize the medications
\end{abstract}


used, with the purpose of facilitating their adherence to treatment, being well accepted by the participant. It is considered that the actions of health education carried out with the individual were able to offer a higher quality of life and control of the disease.

Key words: Systemic Arterial Hypertension. Home visit. University Extension. Quality of life.

\section{INTRODUÇÃO}

As transformações sociais, econômicas e culturais ocorridas na sociedade ao longo do tempo vêm modificando o padrão de vida da população, bem como a forma que elas interagem com a comunidade e seus componentes (BRASIL, 2008). Em alguns casos os novos modelos de consumo social não são bem aceitos ou assimilados pela população, gerando um déficit no autocuidado, repercutindo em alterações e problemas de saúde pública, destacando-se a Hipertensão Arterial Sistêmica (HAS) (MENDES et al., 2016). Mendes e outros autores ainda destacam dados sobre a HAS no seguinte trecho:

Considerado como uma doença crônica, a hipertensão arterial sistêmica acomete aproximadamente $25 \%$ da população mundial, com previsão de aumento de $60 \%$ dos casos dessa doença em 2025. No Brasil, estima-se que $30 \%$ da população, a partir de 40 anos tenha a doença (MENDES et al., 2016, p. 53).

Neste sentido, o controle da HAS está intimamente relacionado às mudanças nos hábitos de vida, assim como a alimentação adequada, a diminuição de sal e o controle do peso, a prática regular de exercícios físicos, o abandono do tabagismo e a redução do uso excessivo de álcool (BRASIL, 2008). Esses fatores precisam ser adequadamente abordados e controlados para que haja os níveis desejados da pressão arterial, fazendose necessário à prática do autocuidado pelo próprio indivíduo. (BALDUINO et al., 2013).

Segundo Moura et al. (2011), indivíduos hipertensos possuem conhecimento sobre os hábitos inadequados, porém os mesmos sentem-se desmotivados para realizarem mudanças no cotidiano, em virtude da cronicidade da doença. Por outro lado, o mesmo autor traz ainda que é possível visualizar possibilidades de mudanças, por meio de condutas que valorizem o outro, seja no ambiente familiar, nas Visitas Domiciliares (VD), ou nas unidades de saúde, promovendo, assim, maior adesão da pessoa hipertensa ao tratamento (MOURA et al., 2011).

Em vista disso, a enfermagem possui como principal preocupação a necessidade de ações de promoção da saúde e no autocuidado dos indivíduos com HAS, oferecendo subsídios contínuos para a sua qualidade de vida (MENDES et al., 2016). O enfermeiro utiliza-se principalmente da VD para organizar tal tarefa, sendo que esta possibilita um contato direto com a realidade do indivíduo e seu contexto familiar e comunitário, estando assim, o profissional munido de estratégias de educação em saúde que ofereçam resolutividade na assistência (BRASIL, 2008). 
Sob este limiar, o objetivo deste estudo é verificar a instrumentalização de práticas de educação em saúde durante visitas domiciliares a um indivíduo com Hipertensão Arterial Sistêmica por meio de um projeto de extensão universitária.

\section{METODOLOGIA}

Trata-se de um estudo de caso, intervencional e com abordagem qualitativa, tendo como participante um indivíduo com diagnóstico de HAS, o qual é atendido pelo projeto de extensão universitária "Ações Interdisciplinares de Cuidado em Saúde", desenvolvido pela Universidade do Vale do Taquari - UNIVATES, que mantém suas ações junto ao bairro Santo Antônio, no município de Lajeado/RS.

A coleta de dados ocorreu durante os meses de março a junho de 2017, período de atuação dos acadêmicos realizaram VD junto ao referido projeto de extensão. As informações coletadas são provenientes do diário de campo dos acadêmicos, sendo que se trata de uma construção da história de saúde e doença do indivíduo, bem como as observações de seu contexto.

As VD possuíram duração média de duas horas cada, sendo que nas ocasiões foram sendo realizadas as anotações com base nos relatos do próprio participante, que desde o inicio aceitou e estava ciente da realização das anotações e coleta de seus dados para posteriores estudos. Neste estudo foram respeitados os preceitos éticos definidos pela Resolução 466 de 2012 do Conselho Nacional de Saúde, sendo utilizada a sigla DS para identificar o participante e manter em sigilo sua identidade.

\section{RESULTADOS E DISCUSSÃO}

\section{Breve histórico do participante e seu contexto}

O participante DS, 71 anos, viúvo, aposentado, tabagista há 54 anos, portador de HAS, sofreu um Acidente Vascular Cerebral (AVC) em agosto de 2016 que resultou em diminuição de força em membro superior e inferior direito, biópsia de próstata benigna, faz uso de medicações contínuas. O indivíduo reside sozinho em função de conflitos familiares entre suas filhas, isso faz com que ele esteja passando por um momento complicado de sua vida devido a essa situação. DS foi casado durante 54 anos, sua esposa era portadora de Diabetes Mellitus (DM), colesterol, doença pulmonar granulomatosa, depressão e tabagista, vindo a óbito em setembro de 2016 em sua própria residência.

Buscando compreender melhor as relações e características do grupo familiar, os estudantes construíram uma Genograma Familiar (Imagem 1). DS e sua esposa tiveram nove filhos sendo cinco homens, dentre estes três em óbito e quatro mulheres. 


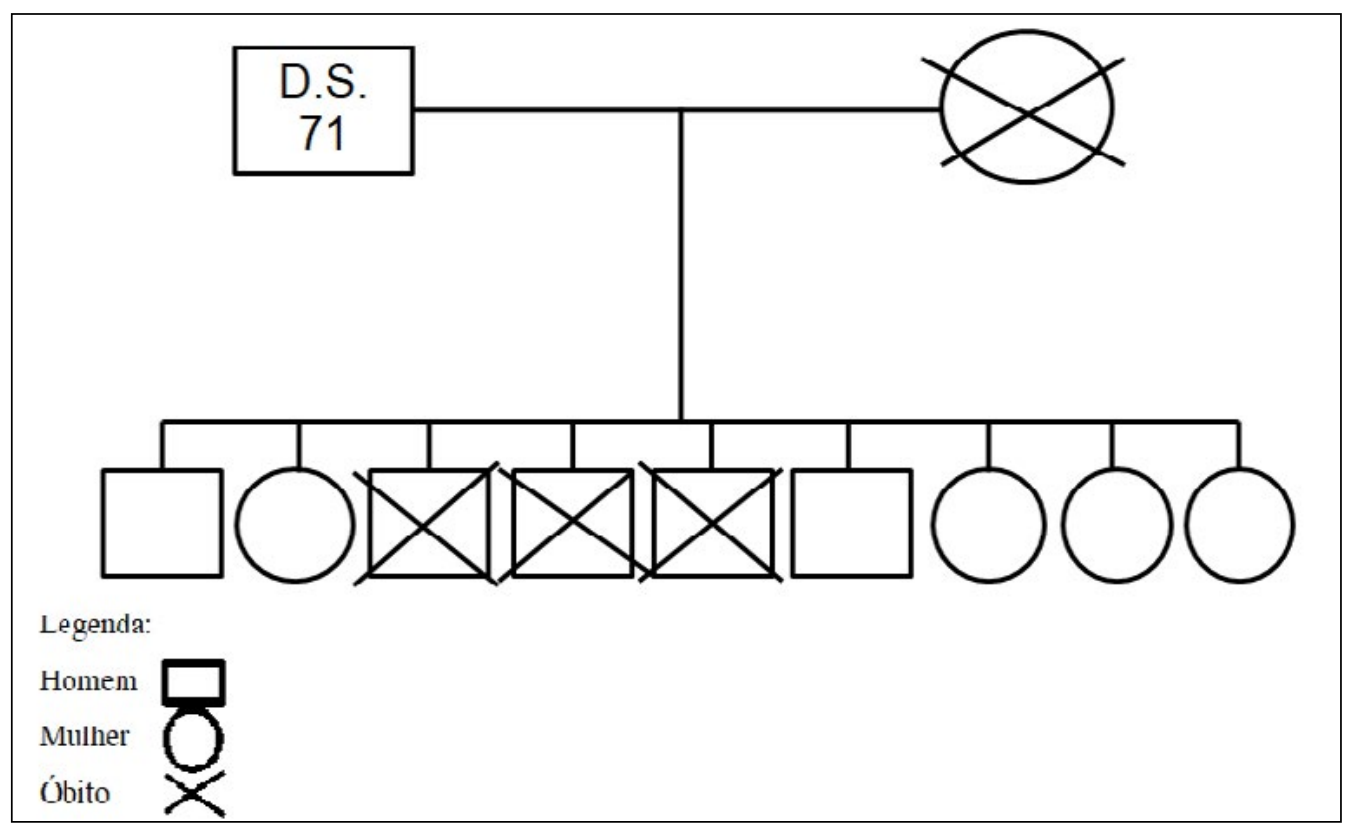

Fonte: Pesquisadores, 2017.

\section{Construção das intervenções de educação em saúde}

Durante o processo de construção das intervenções de educação em saúde, surgiram três eixos principais que recebem ênfase nas categorias a seguir, a saber: Intervenção 01: Hábitos alimentares com ênfase na diminuição de sal $X$ inclusão de frutas e vegetais; Intervenção 02: Prática de exercícios físicos e promoção da saúde; Intervenção 03: Facilitadores do autocuidado com adesão ao tratamento medicamentoso.

\section{Intervenção 01: Hábitos alimentares com ênfase na diminuição de sal X inclusão de frutas e vegetais}

O estilo de vida é claramente um dos grandes responsáveis pela patogenicidade e a elevada prevalência da HAS na sociedade contemporânea, devido principalmente à alimentação rica em sódio (REZENDE et al., 2006). De acordo com o Caderno de Atenção Básica do Ministério da Saúde (BRASIL, 2013), a alimentação tem um papel importante no sucesso do tratamento e na prevenção da HAS, ao passo que a dispensa de certos alimentos na dieta favorecem seu controle.

Conforme Pires, Santos e Pinho (2015), os hábitos alimentares de indivíduos hipertensos devem incluir redução da quantidade de sal e restrição das fontes 
industrializadas, dando preferência a temperos naturais. O consumo elevado de sal está associado aos níveis pressóricos elevados e desenvolvimento de doenças cardiovasculares, em média, a cada redução de $3 \mathrm{~g}$ de sal, há um decréscimo de $5 \mathrm{mmHg}$ na pressão sistólica (PIRES; SANTOS; PINHO, 2015).

Considerando que o consumo de sal no Brasil é alto, e que esse consumo excede os limites máximos recomendados para a sua ingestão, este prejudica ainda mais a saúde de hipertensos. Uma das intervenções que o grupo realizou foi a disponibilização de temperos que podem substituir o sal no seu cotidiano, podendo citar o açafrão, alho, cebola e alecrim, tendo em vista que DS possa reduzir a quantidade de sal em suas refeições. Sobre a redução do sal na alimentação DS relata dificuldades na adesão às orientações, conforme o trecho a seguir:

"Apesar de eu morar sozinho, algumas vezes durante a semana minha filha faz meu almoço, mas ela é muito preocupada com minha alimentação e poupa no sal. Mas isso não é problema, pois levo o meu saleiro para a mesa e acrescendo umas pitadas durante minhas refeiçôes."

Os indivíduos portadores de HAS, devem também evitar o consumo de gorduras e incluir frutas e verduras no plano alimentar diário, com ênfase em vegetais, frutas cítricas e cereais integrais (BRASIL, 2008). Com base nesta afirmativa, durante uma das VD, foi apresentado ao usuário à Pirâmide Alimentar, apontando os alimentos saudáveis em detrimento daqueles que não são recomendados para indivíduos com hipertensão.

Os acadêmicos buscaram investigar os hábitos alimentares durante as VD, identificando a base do consumo alimentar, cujo objetivo foi delinear o estado nutricional e possíveis inadequações alimentares do indivíduo. Neste sentido, a equipe que realiza VD possui um papel fundamental quanto à orientação e acompanhamento do hipertenso na sua área de abrangência, a fim de estabelecer estratégias para o melhor controle de sua pressão arterial (PIRES; SANTOS; PINHO, 2015).

Visando manter um controle sobre os alimentos consumidos, disponibilizou-se um Diário Alimentar, onde o usuário anotou durante uma semana todo o seu roteiro de refeições desde o período da manhã até o período da noite. Tendo como base o diário e a realização de outras $\mathrm{VD}$, percebeu-se a dificuldade para a adaptação do que havia sido orientado nas anteriormente, apesar do indivíduo ingerir verduras em praticamente todas as refeições e frutas pelo menos duas vezes por dia, a diminuição do sal não foi tão satisfatória.

\section{Intervenção 02: Prática de exercícios físicos e promoção da saúde}

Conforme Ministério da Saúde a pratica de atividade física além de melhorar a pressão arterial, também influencia na qualidade do sono e ajuda a combater o estresse 
(BRASIL, 2014). É importante praticar pelo menos 30 minutos em intensidade moderada de algum exercício físico, como caminhada, ciclismo, corrida, ou então o hipertenso pode optar por fazer exercícios com pesos e alongamentos sob orientação de profissionais capacitados, ainda segundo o Caderno de Atenção Básica do Ministério da Saúde, os indivíduos que não praticam atividade física tem risco de 30\% a 50\% de desenvolver hipertensão a mais do que indivíduos que praticam (BRASIL, 2014).

Identificou-se que neste caso o envelhecimento favoreceu o aumento da inatividade física, fator de risco que contribui para o aumento da incidência de doenças crônicas. De fato, o idoso é mais suscetível aos efeitos adversos do sedentarismo, ao exercício físico de intensidade elevada e à terapia medicamentosa, sendo necessária maior compreensão dos efeitos do envelhecimento associados a esses fatores (BRASIL, 2014).

De acordo com esse conhecimento, foram programados alongamentos e exercícios com pesos de areia que foram adaptados para o indivíduo, levando em consideração a sua dificuldade de realizar caminhadas, exercícios que exigem maior força motora e ainda, a diminuição de força devido ao AVC. Neste contexto o hipertenso aderiu bem a essa intervenção, realizando os alongamento e exercícios semanalmente. Relatou melhora na qualidade do sono, diminuição de dor nos membros inferiores, motivação para combater o seu sedentarismo e ocupar-se no seu tempo livre.

\section{Intervenção 03: Facilitadores do autocuidado com adesão ao tratamento medicamentoso}

Muitos hipertensos desenvolvem atitudes que se transformam em fatores facilitadores no desenvolvimento do autocuidado, pois revertem em uma maior possibilidade de controle da pressão arterial, como consequência de uma mudança nos hábitos cotidianos (LOPES et al., 2009). Verificou-se na o participante do estudo se manteve receptivo às intervenções realizadas, o que permitiu a adesão e auxílio em seu autocuidado.

Segundo Lopes et al. (2009), acredita-se que a adesão ao tratamento está atrelada ao papel educacional do profissional ou equipe junto ao usuário, o que se atribui à orientação do autocuidado, além disso, percebe-se a importância da realização de uma assistência integral, através de uma relação empática, solidária e pautada na confiança mútua. É possível perceber as atitudes e práticas que prejudicam o autocuidado, sendo que a ausência de sintomas é uma das principais, pois neste caso a HAS, muitas vezes, é assintomática o que leva o indivíduo a negligenciar sua existência.

Porém segundo o Ministério da Saúde muitos hipertensos apresentam outras doenças associadas trazendo implicações significativas para o gerenciamento das ações terapêuticas necessárias para o controle, já que o tratamento para a HAS exige perseverança, motivação e educação continuada (BRASIL, 2014). Com base nesse 
gerenciamento, é explicito que uma das ações terapêuticas para facilitar a adesão ao autocuidado da HAS, é a utilização correta de suas medicações de uso contínuo.

Conforme Freitas, Nielson e Porto (2015), a adesão imprópria ao tratamento medicamentoso deve ser detectada pelos profissionais de saúde principalmente em relação aos idosos para conhecer os fatores que o levaram a tomar as medidas erradas, para que se possa ajudá-lo. É comum os medicamentos serem utilizados de maneira errônea, não condizendo com a posologia prescrita e ainda, podendo ocasionar alterações de várias formas, como a redução de benefícios, o aumento dos riscos, ou ambos (FREITAS; NIELSON; PORTO, 2015).

Nesta intervenção, levando em conta a importância da adesão em seu próprio autocuidado, foi realizado com o usuário durante algumas VD, explicações sobre a utilização dos seus medicamentos, frisando a indicação, os horários, as doses e os possíveis eventos adversos, assim como, os benefícios dos mesmos se utilizados da maneira correta.

Visto a dificuldade do entendimento sobre esse assunto e levando em consideração o ambiente familiar em que DS se encontra, foi optado por criar uma caixa organizadora de medicamentos, com um espaço adequado para fácil compreensão referente aos seus medicamentos. Nas VD seguintes foram revisadas as medicações usadas em todos os turnos, após as trocas de informações, percebemos que houve um bom retorno de adesão com a nova forma de organização, facilitando o uso de suas medicações.

\section{CONSIDERAÇÕES FINAIS}

Com este estudo realizou-se um levantamento das necessidades de um indivíduo hipertenso, podendo-se assim mapear um plano de ações direto no domicilio, onde a equipe utilizou-se da educação em saúde como ferramenta assistencial resolutiva. Considera-se que mesmo DS não seguindo o tratamento medicamentoso corretamente, continuar com o consumo de sal em suas refeições, é de extrema importância que a equipe se integre da realidade do indivíduo, e através disso propor intervenções que possam ajudá-lo, levando em considerações as suas patologias e suas restrições.

Compreende-se que a HAS representa um problema de saúde potencial para a diminuição da qualidade de vida do indivíduo, no entanto verificou-se que se a mesma for manejada corretamente eleva substancialmente a chance se seus sintomas serem controlados. Para que isso aconteça infere-se que os profissionais da área da saúde saibam identificar e reverter situações como aquela trabalhada neste estudo, utilizandose da educação em saúde frente ao processo de melhoria da saúde da população.

Verificou-se que as intervenções realizadas no projeto ajudaram o usuário a ter uma melhora na qualidade de sua vida, propondo modificações em seu cotidiano que influenciam diretamente no modo de agir frente à doença. Sendo assim, todos os resultados apresentados nesse estudo demonstraram a necessidade de realização de 
educação em saúde por meio destas intervenções realizadas, que contribuíram para a melhora da qualidade de vida do indivíduo.

\section{REFERÊNCIAS}

BALDUINO, A. F. A. et al. Análise conceitual de autogestão do indivíduo hipertenso. Revista Gaúcha de Enfermagem, v. 34, n. 4, p. 37-44, 2013.

BRASIL. Ministério da Saúde. Diretrizes e Recomendações para o Cuidado Integral de Doenças Crônicas Não-Transmissíveis. Brasília-DF. 2008.

BRASIL. Ministério da Saúde. Secretaria de Atenção à Saúde. Departamento de Atenção Básica. Estratégias para o cuidado da pessoa com doença crônica: hipertensão arterial sistêmica. Cadernos de Atenção Básica, No 37. 2013.

BRASIL, Ministério da Saúde. Secretaria de Atenção à Saúde. Departamento de Atenção Básica. Estratégias para o cuidado da pessoa com doença crônica: hipertensão arterial sistêmica. Cadernos de Atenção Básica, No 37. 2014.

FREITAS, J. G. A.; NIELSON, S. E. O.; PORTO, C. C. Adesão ao tratamento farmacológico em idosos hipertensos: uma revisão integrativa da literatura. Rev soc Bras Clin Med, v. 13, n. 1, p. 75-84, 2015.

LOPES, M. C. L. et al. O autocuidado em indivíduos com hipertensão arterial: um estudo bibliográfico. Revista Eletrônica de Enfermagem, v. 10, n. 1, $2009 .$.

MENDES, C. R. S. et al. Prática de autocuidado de pacientes com hipertensão arterial na atenção primária de saúde. Revista da Rede de Enfermagem do Nordeste, v. 17, n. 1, 2016.

MOURA, D. J. M. et al. Cuidado de enfermagem ao cliente com hipertensão: uma revisão bibliográfica. Revista Brasileira de Enfermagem, v. 64, n. 4, 2011.

PIRES, D. S.; SANTOS, K. C.; PINHO, L. Hábitos alimentares dos hipertensos sob a ótica da enfermagem: revisão integrativa. Revista de enfermagem UFPE, v. 9, n. 5, p. 8453-8460, 2015.

REZENDE, F. A. C. et al. Índice de massa corporal e circunferência abdominal: associação com fatores de risco cardiovascular. Arq Bras Cardiol, v. 87, n. 6, p. 728-34, 2006. 